The new BioImaging column of BioTechniques will feature short articles devoted to microscopy and digital imaging. The subject matter will address details of the methods used to produce images of cells and tissues at any magnification and resolution, and might include "tricks-of-the-trade", novel methods of specimen preparation, practices of image collection, tips on the digital manipulation and publication of images and historical perspectives.

\section{The 39 Steps: A Cautionary Tale of Quantitative 3-D Fluorescence Microscopy}

\author{
Jim Pawley \\ University of Wisconsin-Madison, \\ Madison, WI, USA
}

We all know that fluorescent micrographs reveal the location of the labeled molecules in a tissue, right? Well, maybe not. In fact, all you can be really sure of measuring with most laser-scanning confocal microscopes in the fluorescence mode is some feature of the number of photons collected at a particular time. We can hope this is an accurate measure of one or two interesting parameters - the local analyte concentration or the local ion concentration. In fact, many factors affect the numerical values actually stored in the computer memory at any given moment. Over the years, students taking The 3D Microscopy of Living Cells course held each June at the University of British Columbia, compiled a list of these extraneous factors. In the first year, the list grew to 39 entries, and so we borrowed the name of the Alfred Hitchcock film for our list. Since then, the list has continued to grow! (More information on the course can be found at www.cs.ubc.ca/spider/ladic/course/bulletin.html).

Although this article can't fully describe each term, brief and useful explanations are included. The terms appear in bold lettering and many interact with other terms in bold lettering. Note that many of these variables are usually thought of in terms of their effect on spatial resolution. They are listed here because reduced resolution translates into "putting the same number of exciting photons into a larger spot" This lowers the excitation intensity - the number of photons produced by a given molecule-and the fraction of these that are detected. It is often forgotten that normal signal levels in fluorescence confocal microscopy correspond to only 10-20 photons/pixel in the brightest areas. Under such conditions, statistical noise is a more important limitation on spatial resolution than that defined by the Abbé equation (1).

A flow diagram of a generic laser scanning confocal microscope with the locations of many of the " 39 " features mentioned in the text is shown in Figure 1.
The laser unit (Figure 1A) is the illumination source, and in general, the fluorescence measured is proportional to the laser power level. Although total laser output power is usually regulated, the amount of power in each line of a multiline laser may not be, and may vary widely with time. The wavelength affects optical performance, and through the absorption spectrum of the dye, it determines the amount of fluorescence produced.

Power output instability is usually noise; its instability is usually less than $1 \%$, but lasers can become increasingly unstable as they age. Because dust, misalignment or mechanical instability can cause random changes of $10-30 \%$, the efficiency of the optical coupling to the connecting fiber (if used) is critical.

The alignment and reflection characteristics of laser mirrors can be the source of long-term drift in laser output. Since the source of the laser light is determined by the laser mirrors, beam-pointing error/alignment is important. Instability here will show up as changes in brightness because changes in the apparent source position will alter the efficiency of the optics coupling the laser light into the single-mode optical fiber used in most instruments.

The numerical aperture of the objective lens (Figure 1B) affects the fraction of the light emitted by the specimen that can be collected. This is also true for light from the laser.

Objective magnification is inversely related to the diameter of the objective lens entrance pupil. The objective will only function properly if the entire entrance pupil is filled with exciting laser light. Underfilling will reduce spatial resolution and the peak intensity. Overfilling will cause some laser light to strike the metal mounting of the objective and be lost, also reducing the intensity of the spot.

Cleanliness is important and dirty optics produce much larger and dimmer spots. Transmission (the fraction of light incident on the objective that can be focused into a spot on the other side of it) varies with wavelength. Beware of using some relatively new, multicoated optics in IR range. Chromatic and spherical aberration both make the spot bigger, and vary with wavelength. In addition, spherical aberration varies strongly with the coverglass thickness and the refractive index of the immersion and embedding media.

Diffraction is the unavoidable limit to optical resolution. It effectively enlarges the image of objects smaller than the diffraction limit, making them appear dimmer than they should be.

The scanning system (Figure 1C), and especially the zoom magnification control, determines the size of a pixel at the specimen. For Nyquist sampling, the pixel should be at least two times smaller than the smallest features that you expect to see in your specimen. Assuming a Rayleigh Criterion resolution of $200 \mathrm{~nm}$, the pixels should be less than $100 \mathrm{~nm}$. Larger ones produce undersampling, reducing the recorded brightness of small features (2).

The effects of the bleaching rate are proportional to the square of the zoom magnification. As for scan speed, the longer the dwell time on a particular pixel, the more signal will be detected and the less it will be distorted by Poisson Noise (3). At high scan speeds (less than $100 \mathrm{~ns} /$ pixel), signals from dyes with fluorescent decay constants that are longer than this dwell time can be reduced. 
Raster size - together with the zoom magnification, the number of pixels along the edges of your raster will determine the pixel size. More pixels ( 1024 x 1024 vs. 512 x 512) make undersampling less likely, but mean that one must either spend less time on each pixel by reducing the number of photons collected and increasing Poisson Noise, or take more time to scan the larger image and possibly cause more bleaching. The optics or the scanning mirrors can introduce geometrical distortion that can result in discordance between the shape of the object and the image. The environment is important: vibration and stray electromagnetic fields can be caused by various pieces of equipment, for example, cooling fans. These can cause improper mirror deflections that result in distortions that may vary with time.

Other optics include transmission, which describes a measure of the absence of absorption and reflectance losses in optical components, particularly neutral density or bandpass filters, beamsplitters and objectives. Reflections from air/glass interfaces usually represent lost signal but may appear as bright spots unrelated to specimen structure. Mirror reflectivity can be a strong function of wavelength in the infrared and ultraviolet, and degrades with exposure to humidity and dust.

The coverslip thickness is the least expensive optical component and the most likely to be carelessly chosen. It should be $170 \pm 5 \mu \mathrm{m}$ in thickness. As for immersion oil, its refractive index must be exactly matched to the objective used. This may only occur over a small temperature range, and alternatively, it can be custom-mixed. The focus-plane position is important because a feature slightly above or below the plane of focus will appear dimmer than a feature in the plane of focus. When collecting 3D data, Nyquist sampling must also be practiced in the spacing of $\mathrm{Z}$ planes. Finally, the mechanical drift of the stage can cause the plane of the object actually imaged to change with time.

The concentration of the dye of interest is probably what

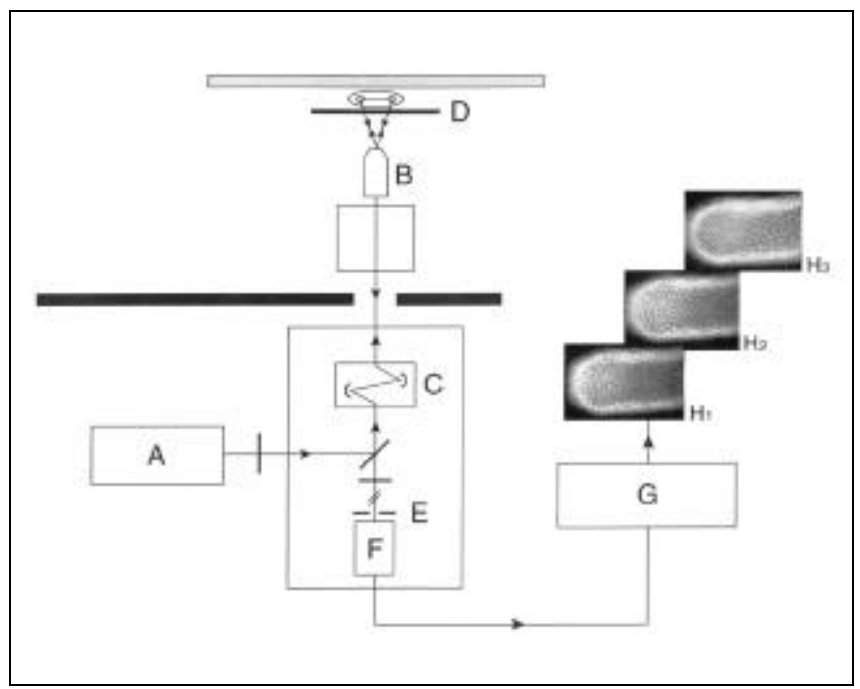

Figure 1. Flow diagram of a generic laser scanning confocal microscope with an inverted light microscope configured for live cell imaging. Individual components are referred to in the text. Three optical sections of a living Drosophila embryo at the cellular blastoderm stage collected at fiveminute intervals are shown $(\mathrm{H} 1, \mathrm{H} 2$ and $\mathrm{H} 3)$. you are trying to measure. Penetration into (or steric exclusion from) the specimen is often a function of ionic strength and $\mathrm{pH}$. The absorption cross-section is a measurement of the fraction of the exciting photon flux that will be absorbed by a dye molecule. It is affected by the method of conjugation to the probe, $\mathrm{pH}$, specific ion concentration and ionic strength.

Quantum efficiency describes the chance that energy absorbed from an exciting photon will be re-radiated as a fluorescent photon. It is a strong function of wavelength, and is also affected by $\mathrm{pH}$, specific ion concentration, ionic strength and dye-protein interactions. Singlet state saturation occurs when greater than one $\mathrm{mW}$ of laser power is used with a highNA objective. Then, the light is intense enough to put most of the dye molecules near the crossover into an excited state, reducing the effective dye quantum efficiency.

Loading refers to the amount of dye you put into your cell. Important variables include the number of dye molecules/antibody or other protein marker, and the fixation/permeabilization protocol used. Quenching is the absorption of the fluorescence emitted from one dye molecule by others nearby. Unloading refers to dye that was in the cell but has now been pumped out or otherwise inactivated.

The substrate-reaction rate applies to dyes with fluorescent properties that are related to their interaction with ions or other molecules in the cell. The pixel-dwell is in the order of microseconds, and so there may not be enough time to reach equilibrium. Prebleaching refers to bleaching of the dye before the present measurement was made.

Compartmentalization is the redistribution of the dye by intracellular processes. Dye/dye interactions refer not only to the quenching just mentioned, but also to fluorescence resonance energy transfer (FRET). This occurs when the emission spectrum of one dye overlaps with the absorption spectrum of a second dye molecule, a few nm away. FRET can result in the emission of light only at the emission wavelength of the second dye. If the second molecule isn't fluorescent as usually happens, the fluorescent light is lost or quenched. The effects of dye on the specimen may affect many environmental variables, including the deterioration of viability of the specimen.

The refractive index of the specimen (Figure 1D) and its embedding medium will determine the severity of the spherical aberration present. Even for viewing aqueous biological specimens using a water objective, the match is unlikely to be perfect or even close. Spherical aberration of this type is the major cause of signal loss with increasing penetration depth.

It is essential to use an immersion oil with the correct refractive index and dispersion (change of refractive index with wavelength). Also, be sure there are no air bubbles that will act as a lens in the middle of it. Check by focusing up and down with the Bertrand lens used for aligning your phase rings.

The vital state of the specimen, for example, dying cells have different shapes, sizes and ionic environments than living ones. Autofluorescence refers to the presence of endogenous fluorescent compounds. The efficiency of these molecules may vary with $\mathrm{pH}$, ionic concentration and metabolic state. Additional autofluorescence can be produced by improper fixation, particularly when using glutaraldehyde.

Refractile structures or organelles between the objective and the plane of focus, for example, spherical globules of 
lipid, may refocus the beam to a totally unknown location. Smaller features may have lesser effects but any refractile features that scatter light out of the beam will reduce the intensity of the excited spot and hence, of the detected signal. Their cumulative effect also adds up to cells having an RI much higher than that of water.

The presence or absence of highly stained structures above or below the focus plane refer to the Z-resolution that is finite. Large structures also may absorb substantial exciting light if highly stained.

The signal that passes through the pinhole (Figure 1E) is proportional to the square of the diameter of the pinhole (or its size), which is usually set to be equal to the diameter of the Airy Disk at the plane of the pinhole. Alignment is important and the image of the laser that is focused onto the specimen and then refocused back through the optical system should coincide with the center of the pinhole.

The detector (Figure 1F) is usually a photomultiplier tube or PMT. The detected signal is directly proportional to the quantum efficiency $(\mathrm{QE})$. The effective $\mathrm{QE}$ of the PMT tubes used in most confocals drops from about $15 \%$ in the blue end to approximately $4 \%$ in the red end of the spectrum. As for response time: most fluorescent signals can be amplified rapidly, but detectors for others, such as transmembrane currents re- spond slowly, making slow scanning speeds necessary.

The PMT voltage determines the amplification of the PMT. An increase of $50 \mathrm{~V}$ corresponds to a factor of about two more in gain. Beware that PMT black level or (brightness) control permits the addition or subtraction of an arbitrary amount from the signal presented to the digitizer. The black level should be set so that the signal level in the darkest parts of the image is 5-10 digital units.

There are many possible sources of noise and all will distort the recorded value. Usually, PMTs produce dark current noise that is small compared to the signal level. However, this is less true for red-sensitive PMTs that are allowed to become warm or when viewing poorly stained specimens. Apart from any stray light that may inadvertently reach the PMT, the main remaining noise source is Poisson or statistical noise. This is equal to the square root of the number of photons recorded in a given pixel. The result is it becomes larger at higher signal levels, even though the ratio of signal to noise improves.

Digitization, usually using a computer (Figure 1G) should be linear. The electronic signals presented to the digitizer of "8-bit" microscopes must be of a size to be recorded between 1 and 255. Because of statistical noise, a value between 10 and 220 is safer. The digital conversion factor refers to the ratio between the number of photons detected and the number stored. This depends on the PMT voltage and other electronic gain, but is usually about 30 for normal specimens recorded on 8-bit instruments.

Remember, to measure one of these factors accurately, one must hold the other 38 constant. Good luck!

\section{ACKNOWLEDGMENTS}

I would like to thank Leanne Olds and Steve Paddock, Dept. Molecular Biology, University of Wisconsin for Figure 1 and William Russin, Department of Plant Pathology, University of Wisconsin and Steve Paddock for the cover illustration.

\section{REFERENCES}

1.Inoue, S. 1995 Foundations of confocal scanned imaging in light microscopy, p. 1-14. In J.B. Pawley (Ed.), Handbook of Biological Confocal Microscopy, 2nd. ed. Plenum Press, New York.

2.Webb, R. H. and C.K. Dorey. 1995. The pixilated image, p 55-66. In J.B. Pawley (Ed.), Handbook of Biological Confocal Microscopy. 2nd. ed. Plenum Press, New York.

3.Sheppard, C J.R., X. Gan, M. Gu and M. Roy. 1995. Signal-to-noise in confocal microscopes, p 363-370. In J.B Pawley (Ed.), Handbook of Biological Confocal Microscopy, 2nd. ed. Plenum Press, New York.

Address correspondence to Dr. Jim Pawley, Department of Zoology, University of WisconsinMadison, 1525 Linden Drive, Madison, WI 53705, USA. Internet: jbpawley@facstaff.wisc.edu

Suggestions for contributions to the Biolmaging feature are welcomed by its editor, Dr. Steve Paddock (paddock@facstaff.wisc.edu) 